\title{
Deuterium-exchange metabolomics identifies $N$-methyl lyso phosphatidylethanolamines as abundant lipids in acidophilic mixed microbial communities
}

\author{
Curt R. Fischer - Paul Wilmes - Benjamin P. Bowen • \\ Trent R. Northen · Jillian F. Banfield
}

Received: 3 April 2011 / Accepted: 23 July 2011

(C) Springer Science+Business Media, LLC 2011

\begin{abstract}
Natural microbial communities are extremely diverse and contain uncharacterized but functionally important small molecules. By coupling a deuterium (D) labeling technique to high mass accuracy untargeted liquid chromatography-electrospray ionization-mass spectrometry (LC-ESI-MS) metabolomic analysis, we found that natural acidophilic microbial biofilms dominated by bacteria of the genus Leptospirillum contained unusual lyso phosphatidylethanolamine (PE) lipids in high abundance (more than $10 \mathrm{nmol} / \mathrm{mg}$ of dry biomass). The unusual polar
\end{abstract}

Curt R. Fischer and Paul Wilmes contributed equally to this work.

Electronic supplementary material The online version of this article (doi:10.1007/s11306-011-0344-x) contains supplementary material, which is available to authorized users.

C. R. Fischer · P. Wilmes · J. F. Banfield ( $\square)$

Department of Earth and Planetary Science, University

of California, Berkeley, Berkeley, CA 94720, USA

e-mail: jbanfield@berkeley.edu

Present Address:

C. R. Fischer

Ginkgo Bioworks, Boston, MA, USA

Present Address:

P. Wilmes

Department of Environment and Agro-biotechnologies, Public Research Centre-Gabriel Lippmann, and Luxembourg Centre for Systems Biomedicine (LCSB), University of Luxembourg, Belvaux, Grand-Duchy of Luxembourg

B. P. Bowen - T. R. Northen

Life Sciences Division, Lawrence Berkeley National Laboratory,

1 Cyclotron Road, Berkeley, CA 94720, USA

J. F. Banfield

Department of Environmental Science, Policy and Management, University of California, Berkeley, CA 94720, USA head group structure of these lipids is similar to lipids found in phylogenetically unrelated acidophilic chemoautolithotrophs and may be related to the affinity of these lipids for iron and calcium ions. Correlations of lyso phospholipid and proteome abundance patterns suggest a link between the lyso phospholipids and the UBA-type substrain of Leptospirillum group II. By combining untargeted metabolomics with $\mathrm{D}$ exchange we demonstrate the ability to identify cryptic but biologically functional small molecules in mixed microbial communities.

Keywords Deuterium exchange $\cdot$ Untargeted metabolomics $\cdot$ Microbial community $\cdot$ Lyso lipid

\section{Introduction}

Chemoautolithotrophic mixed-species microbial biofilms thrive in the very low $\mathrm{pH}$, iron-rich acid mine drainage solutions that exist within the Richmond Mine near Iron Mountain, CA. Similar communities occur at miningrelated sites and bioleaching systems around the world. The Richmond Mine microbial communities have been the subject of extensive metagenomic (Tyson et al. 2004), whole-community shotgun proteomic (Ram et al. 2005; Lo et al. 2007; Denef et al. 2010), metabolomic, biochemical (Singer et al. 2008; Jeans et al. 2008), and structural characterization (Wilmes et al. 2009). Annotated, nearcomplete genome assemblies are available for three lineages of the predominant Leptospirillum bacteria (Goltsman et al. 2009) and also for many of the coexisting archaea, including Ferroplasma acidarmanus (Allen et al. 2007), many related Thermoplasmatales Euryarchaeota, as well as three lineages of nanoarchaea (Baker et al. 2010). These organisms are all difficult to cultivate and most have 
not yet been obtained in pure culture. In addition, the proteomes of these mixed microbial communities include numerous proteins of unknown function, and thus these communities may harbor undiscovered metabolic transformations. Thus, AMD biofilms provide an ideal test bed for applying untargeted strategies for metabolite detection and characterization.

Leptospirillum-dominated biofilms growing in solutions containing near molar concentrations of dissolved metals, especially iron, at $\mathrm{pHs}$ as low as 0.3 can oxidatively dissolve pyrite $\left(\mathrm{FeS}_{2}\right)$ and other sulfide minerals. These conditions are much harsher than those preferred by the workhorse organism of bioleaching studies, Acidithiobacillus ferrooxidans. In contrast to A. ferrooxidans, comparatively little is known about the lipid biology of the Leptospirilla. In this study, we apply a coupled isotopic-labelling/untargeted metabolomics strategy to isolate hydrophobic metabolites from Leptospirillum-dominated biofilms for which extensive biological (metagenomic, proteomic), physical, and chemical data are available. In addition to providing new insights into ecosystem functioning, we report a method that can be more generally applied to characterize small molecules in other biological systems.

Despite the creation and ongoing curation of genomelinked, annotated mass-spectral databases of metabolites, many molecular species detected in studies of a variety of ecosystems do not match any chemical elemental formula in databases, including PubChem (Baran et al. 2010). The high resolving power, sensitivity and mass accuracy achieved in liquid chromatography-electrospray ionizationquantitative time-of-flight mass spectrometry (LC-ESIqTOF-MS) provides a basis for constraining the chemical formula and structure of detected spectral features, but unique chemical identities are hard to discern from the mass information alone. Heuristic rules based on stoichiometric restrictions and/or natural isotopologue (Kind and Fiehn 2006, 2007) abundances can help, as can stable isotope labeling (Baran et al. 2010), most frequently done with nitrogen $\left({ }^{15} \mathrm{~N}\right)$ or carbon $\left({ }^{13} \mathrm{C}\right)$. Here we apply a technique based on post-extraction deuterium (D) labeling of metabolites (Liu et al. 2001, 2007) to the elucidate abundant metabolic features detected in a model mixedspecies microbial community.

\section{Materials and methods}

2.1 Acid mine drainage field site and biofilm sample collection

Samples were collected within the Richmond Mine at Iron Mountain in northern California, frozen on dry ice at the time of collection and kept at $-80^{\circ} \mathrm{C}$ until metabolite extraction. Many samples studied in this paper have been previously described, including the $\mathrm{AB}$ Front and $\mathrm{AB}$ End samples (Ram et al. 2005), the C $+75 \mathrm{~m}$ sample (Wilmes et al. 2009), the B-drift series of samples (Mueller et al. 2010), and the AB Muck Dam DS2 and AB Muck Dam Friable samples (Wilmes et al. 2010).

\subsection{Bioreactor cultivation of representative mixed AMD biofilms}

Representative biofilms were grown in a cultivation apparatus constructed as described previously (Belnap et al. 2010), with some modifications. 9K-BR medium was modified to contain $400 \mathrm{mM} \mathrm{FeSO}_{4}$ as the electron donor, and either $>98$ atom\% $\mathrm{N}\left({ }^{15} \mathrm{NH}_{4}\right)_{2} \mathrm{SO}_{4}$ or natural-abundance $\left(\mathrm{NH}_{4}\right)_{2} \mathrm{SO}_{4}$ was used as the sole nitrogen source. Fresh bioreactors were started by mixing fresh $9 \mathrm{~K}-\mathrm{BR}$ medium with uncentrifuged spent bioreactor medium in a 99:1 ratio by volume. Medium flow rates in all experiments were $0.2-0.3 \mathrm{ml} / \mathrm{min}$.

For isotopic labeling experiments, biofilms were initially grown with medium recycling, as originally described (Belnap et al. 2010). To ensure virtually complete isotopic labeling after biofilm establishment, a second batch of isotopically labeled medium was prepared and flowed through the reactor in a single-pass configuration.

Within the bioreactors, biofilm tens of $\mu \mathrm{m}$ thick grows at the air solution interface. Biofilm samples were excised and stored at $-80^{\circ} \mathrm{C}$ until metabolite extraction.

\subsection{Metabolite extraction and LC-ESI-qTOF-MS analysis}

\subsubsection{Natural biofilm samples}

Approximately $500 \mathrm{mg}$ of frozen biomass $\left(-80^{\circ} \mathrm{C}\right)$ was removed from each sample and analyzed by LC-ESIqTOF-MS as described previously (Wilmes et al. 2010). Technical replicates were extractions done in parallel on multiple $500 \mathrm{mg}$ portions of a given sample. See the Supplemental Methods for full details.

\subsection{Modifications to LC-MS methods for deuteurium exchange experiments}

For D exchange, samples were resuspended in 4:1 acetonitrile:water (v:v) or in 4:1 acetonitrile:D oxide. Polar head group standards were dissolved in either $100 \%$ water or $100 \%$ D oxide. Mobile phase A for D-exchange experiments was either $0.1 \% d_{4}$-acetic acid in D oxide. Mobile phase $\mathrm{B}$ was $d_{4}$-acetic acid in isotopically unlabelled 
acetonitrile. D oxide was $>99.9 \%$ atom $\% \mathrm{D}$ and $d_{4}$-acetic acid was $>99.93$ atom $\% \mathrm{D}$, both from Cambridge Isotope Laboratories, Andover, MA.

The mass accuracy of qTOF-type instruments is improved to nearly $1 \mathrm{ppm}$ by continuous calibration to internal standards co-infused into the ion source. The manufacturer-recommended calibrant solution contains labile protons. To avoid dilution of sample-derived labile deuterons with labile protons from the calibrant solution, internal mass calibration was deactivated while analyzing deuterated samples. Mass accuracy stayed better than $10 \mathrm{ppm}$ under these conditions, as long as the spectrometer had been freshly calibrated immediately before deactivation of internal calibration.

Samples used for growth stage- or morphology-dependent profiling were extracted in triplicate (B series samples) or quintuplicate (AB Muck Friable (ABMF)and $A B$ Muck DS2 samples).

\subsection{Analysis of lipids standards}

Stocks of lipid standards were dissolved in a 5:10:4 mixture of chloroform:methanol:water with $0.1 \mathrm{v} / \mathrm{v} \%$ formic acid. Iron(III) sulfate, iron(II) sulfate heptahydrate, and calcium chloride salts were dissolved in $0.1 \mathrm{v} / \mathrm{v} \%$ formic acid in water. Different combinations of a single lipid type and either iron or calcium salts were diluted to final concentrations of $100 \mu \mathrm{M}$ in a 5:10:4 mixture of chloroform:methanol:water with $0.1 \mathrm{v} / \mathrm{v} \%$ formic acid as solvent. MS analysis was carried out by direct infusion with 5:10:4 mixture of chloroform:methanol:water as the solvent.

\subsection{Estimation of absolute abundance for lyso phospholipids and comparison to diacyl lipid abundance}

Absolute quantification of the lyso lipids in the bioreactorderived AMD biofilm was made by spiking between 7.6 and $253 \mathrm{nmol}$ of 18:0 lyso PE (phosphatidylethanolamine, Avanti Polar Lipids) into lyophilized biomass samples before extraction. To ensure complete lipid extraction, a lipid-specific extraction protocol using a 2:1 methanol:chloroform mixture. Extracts were analyzed by LCMS as described in the Supplemental Methods and the intensity ratios of the MS2 fragments of the internal standard (341 Da) was compared to the MS2 fragment of the isobaric natural lipid (327 Da). Full details are in the Supplemental Methods.

\subsection{Data analysis and bioinformatics clustering}

Custom software was used to identify mass spectral peaks, align peaks across multiple files, and to quantify the peak intensity. A variety of bioinformatics software packages were used to cluster identified peaks and their sample of origin and to construct correlation networks between metabolites, as described in the Supplemental Methods.

\section{Results}

3.1 Metabolomic profiling of acid mine drainage biofilms

Eleven samples of acid mine drainage biofilms (with between 1 and 9 technical replicates for each) were selected for untargeted metabolomic profiling. These samples were chosen because of extensive previous proteomic and geochemical characterization and because they span a range of development stages (DS), morphologies and community compositions.

Untargeted metabolomic profiling of metabolite extracts using reverse-phase C18 column chromatography resulted in identification of 13,291 mass spectral features. Most of these features eluted in the solvent front or shortly thereafter (retention times $<600$ s). 3,059 features with retention times $>600 \mathrm{~s}$ were selected for further analysis. Features were sorted and ranked by their median intensity in the 11 samples, as shown in Fig. 1a. The ten most intensely detected features, and 17 of the 30 most intense features, were found in the mass range between 452 and 483 Da.

Kendrick mass defect analysis (where the mass of the ${ }^{12} \mathrm{CH}_{2}$ moiety is set to 14.00000 ), shown in Fig. 1b, revealed that the features in this mass range were members of a six-member class of compounds, differing by $\pm \mathrm{H}_{2}$ and/or $\pm \mathrm{CH}_{2}$. Several peaks arising from natural-abundance of ${ }^{13} \mathrm{C}$ isotopologues of these features were also among the most abundant of these features, further reinforcing the relative abundance of these features. Figure 1c shows extracted ion chromatograms for representative samples of these features, showing clear chromatographic separation of the different $\mathrm{m} / \mathrm{z}$ values. However, peak shapes for some of the features suggest they consist of isomers.

Accurate mass and relative isotopologue abundance information for this family of lipids showed that the neutral empirical formula of these features was $\mathrm{C}_{n} \mathrm{H}_{2 n} \mathrm{NO}_{7} \mathrm{P}$ and $\mathrm{C}_{n} \mathrm{H}_{2 n}+{ }_{2} \mathrm{NO}_{7} \mathrm{P}$, where $n$ was 21,22 or 23 . $\mathrm{MS}^{2}$ fragmentation patterns from the environmental extracts for the lightest $\mathrm{C}_{n} \mathrm{H}_{2 n} \mathrm{NO}_{7} \mathrm{P}$ lipid $(n=21)$ showed a characteristic neutral loss of $141 \mathrm{Da}$ from the parent ion, indicative of a PE moiety, but heavier members of the class showed neutral losses of $155 \mathrm{Da}$ in addition to $(n=22)$ or in place of $(n=23)$ the PE neutral loss, suggestive of a methyl 
(a)

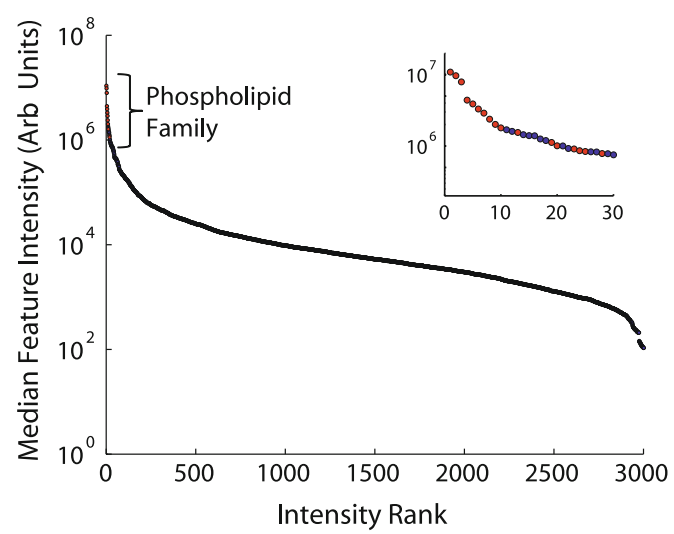

(b)

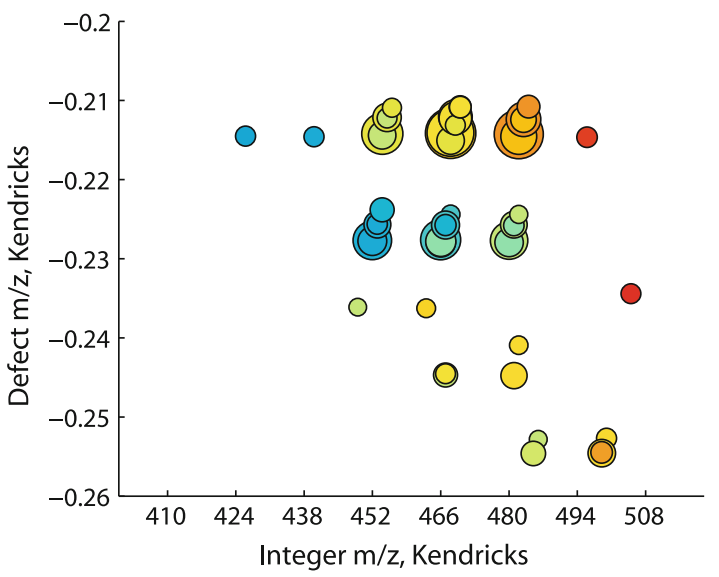

(c)

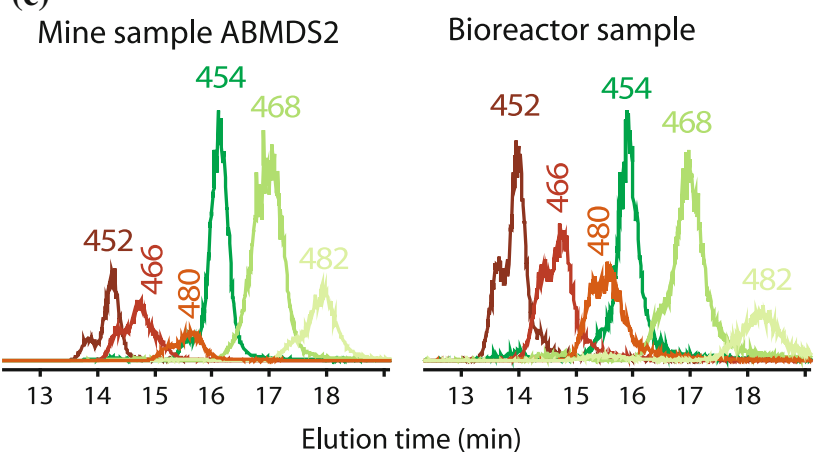

$\left(\mathrm{CH}_{3}\right)$ replacement for hydrogen $(\mathrm{H})$ on these features. However, the location of the methylation could not be definitively assigned on the basis of $\mathrm{MS}^{2}$ information alone. The $n=23$ feature's parent ion mass differed from the $n=21$ feature by $2\left(\mathrm{CH}_{2}\right)$, but only one of these methyl equivalents seemed to be located in the PE headgroup.

\subsection{Stable isotope labeling and $\mathrm{D} / \mathrm{H}$ exchange for de novo metabolite identification}

We hypothesized that a $\mathrm{D} / \mathrm{H}$ exchange method, similar to one previously reported by Liu and co-workers (2001) could discriminate between structural isomers-in this case, $\mathrm{N}$-methylation states - and would provide a simple
4 Fig. 1 Detection of highly abundant intact polar lipids of unknown identity in samples of acidophilic, mixed-species biofilms. a A rankabundance plot of spectral features found through multi-sample metabolomic analysis of an acidophilic mixed-species biofilm community. The median intensity for each feature in the 37 samples is plotted against the rank of the median intensity. Features corresponding to empirical formulas of $\mathrm{C}_{n} \mathrm{H}_{m} \mathrm{NO}_{7} \mathrm{P}(n=21,22,23 ; m=42,44$, $46,48)$ are shown in red; other features are in blue. Inset: a closer view of the most intensely detected metabolic features. b A Kendrick mass defect diagram showing the median intensity (dot size), retention time (color), and mass interrelationships between detected between $\mathrm{C}_{n} \mathrm{H}_{m} \mathrm{NO}_{7} \mathrm{P}$ phospholipids. Horizontally spaced dots indicate features whose mass difference exactly corresponds to $\pm \mathrm{CH}_{2}$. c Extracted ion chromatograms for the six most abundant members of the $\mathrm{C}_{n} \mathrm{H}_{m} \mathrm{NO}_{7} \mathrm{P}$ phospholipid family. Elution patterns show two groups of three subfamilies, one at $\mathrm{m} / \mathrm{z}$ values of $452.2772,466.2928$, and $480.3084 \mathrm{Da}$, and another at 454.2928, 468.3084, and $482.3241 \mathrm{Da}$. Each compound differs from its subfamily members by $\pm \mathrm{CH}_{2}$ and from the other family by $\pm \mathrm{H}_{2}$ (Color figure online)

experimental approach to gather additional structure information on these lyso PE lipids. This methodology is schematized in Fig. 2. It relies on coupling (i) complete exchange of labile metabolite protons with deuterons and (ii) MS, especially high-resolution MS, to determine the number of labile protons present in particular metabolites. The microflow $(<100 \mu \mathrm{l} / \mathrm{min})$ chromatography conditions employed enable economic use of completely $(>99.9$ atom\% D) deuterated solvents to be used for sample reconstitution and chromatography, as shown in Fig. 2a. Each metabolite extract can thus be analyzed in protonated and deuterated forms. Figure $2 \mathrm{~b}$ and $\mathrm{c}$ show that reconstitution in this way leads to virtually complete exchange of labile protons for deuterons in the case of two model metabolites: the hydrophobic 3-(decyldimethylammonio)propanesulfonate and the polar dipeptide glycylglycine. In contrast, techniques based on in-source introduction of $\mathrm{D}_{2} \mathrm{O}$ or other gases with labile deuterons do not achieve such complete exchange, as shown by the bottom (purple) mass spectra for the two sample compounds analyzed by D-exchange.

Figure 3a shows examples of the results of D exchange studies of two of the six abundant phospholipids. Ion chromatograms extracted from LC-MS data at masses corresponding to substitution of zero to six exchanged protons for deuterons on the $\mathrm{m} / \mathrm{z}=452$ feature (with an empirical formula of $\mathrm{C}_{21} \mathrm{H}_{42} \mathrm{NO}_{7} \mathrm{PH}^{+}$) showed significant intensity only for five deuteron substitutions. The D-exchanged $\mathrm{C}_{21} \mathrm{H}_{42} \mathrm{NO}_{7} \mathrm{PH}^{+}$lipid thus corresponds to the formula $\mathrm{C}_{21} \mathrm{H}_{38} \mathrm{D}_{4} \mathrm{NO}_{7} \mathrm{PD}^{+}$. In contrast, ion chromatograms extracted similarly for the D-exchanged $\mathrm{C}_{23} \mathrm{H}_{46}$ $\mathrm{NO}_{7} \mathrm{PH}^{+}$feature showed high intensity for only four deuteron substitutions, or an ion formula of $\mathrm{C}_{23} \mathrm{H}_{43} \mathrm{D}_{3}$ $\mathrm{NO}_{7} \mathrm{PD}^{+}$.

As shown in Fig. $3 \mathrm{~b}$ and $3 \mathrm{c}, \mathrm{MS}^{2}$ fragmentation studies were conducted on D-exchanged lipids. When fragmented 


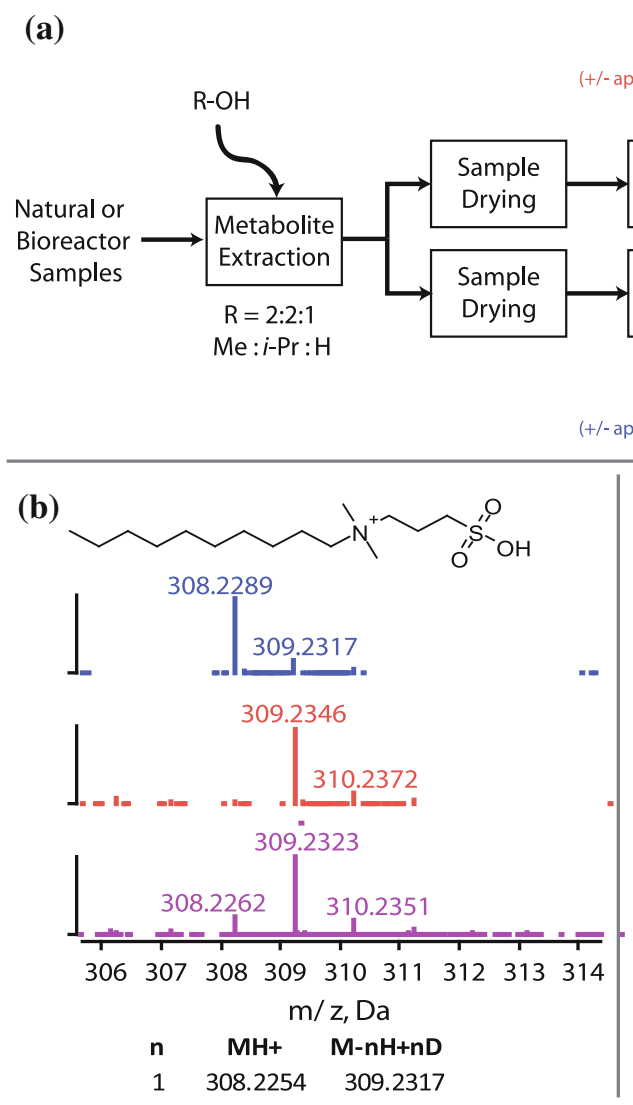

Fig. 2 D-exchange metabolomics. a Sample handling workflow. Metabolite extracts are split into two aliquots and dried under vacuum. One aliquot is reconstituted and chromatographed in protic solvents with a natural abundance of ${ }^{1} \mathrm{H}$. The other is reconstituted and chromatographed in deuterated solvents, where $>99 \%$ of the labile solvent protons are replaced with ${ }^{2} \mathrm{H}$. In ESI-MS, labile protons can exchange with analytes by gas phase transfer, if desired. b D-exchange mass spectrum of decyldimethylammoniopropane sulfonate. Protonated $\left(\mathrm{MH}^{+}\right)$molecules contain one exchangeable $\mathrm{H}$ each. The mass spectrum in protonated solvents (top, blue) is thus downshifted from the mass spectrum in $>99 \%$ deuterated solvents

by collision-induced dissociation, the $\mathrm{m} / \mathrm{z}=452$ lipid showed fragments at 44, 62, 311, and $452 \mathrm{Da}$. The mass of each of these fragments was shifted by exchange of deuterons for protons in the corresponding $\mathrm{MS}^{2}$ spectrum of the D-exchanged sample. The fragments at 44 and $62 \mathrm{Da}$ contained 2 and 4 exchangeable hydrogens, respectively. $\mathrm{MS}^{2}$ fragmentation analysis was repeated on biofilm communities grown in the laboratory with ${ }^{15} \mathrm{NH}_{4}{ }^{+}$as the sole nitrogen source, which resulted in a $0.997 \mathrm{Da}$ increase in the location of the fragments at 44 and $62 \mathrm{Da}$, indicating that these fragments contain one nitrogen (data not shown). These data were consistent with an ethanolamine moiety, and analysis of authentic ethanolamine by CID MS ${ }^{2}$ also gave rise to identical fragments at 44 and $62 \mathrm{Da}$, as shown in Supplemental Fig. S1. Thus, all mass spectra and stable isotope labeling experiments for the $\mathrm{m} / z=452$ lipid were

consistent with the structure shown at right in Fig. 3b. The $m / z=480$ has a parent mass that is $\mathrm{C}_{2} \mathrm{H}_{4}$ higher than the $m / z=452$ lipid, a mass difference which commonly arises from $\mathrm{C}_{2}$ chain extension reactions in fatty acid biosynthesis. However, as shown in Fig. 3c, D exchange experiments on the $m / z=480$ lipid revealed that the $\mathrm{C}_{2} \mathrm{H}_{4}$ mass difference resulted from two distal substitutions of $\mathrm{CH}_{3}$ for $\mathrm{H}$. One substitution of $\mathrm{CH}_{3}$ for $\mathrm{H}$ displaced a $\mathrm{H}$ on the headgroup-derived fragments, leading to fragments at 58 and 76. $\mathrm{D}$ exchange experiments showed that the $\mathrm{H}$ replaced had been exchangeable because D-exchanged fragments showed the fragments at 58 and 76 had one and three exchangeable hydrogens, respectively, compared to two and four exchangeable hydrogens found in Fig. $3 \mathrm{~b}$ for the fragments at 44 and $62 \mathrm{Da} .{ }^{15} \mathrm{~N}$ labeling of laboratorygrown biofilm showed that the 58 and 76 fragments each 
(a)

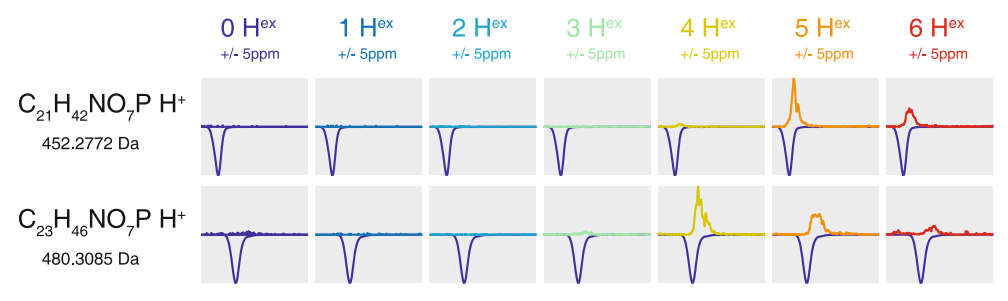

(b)
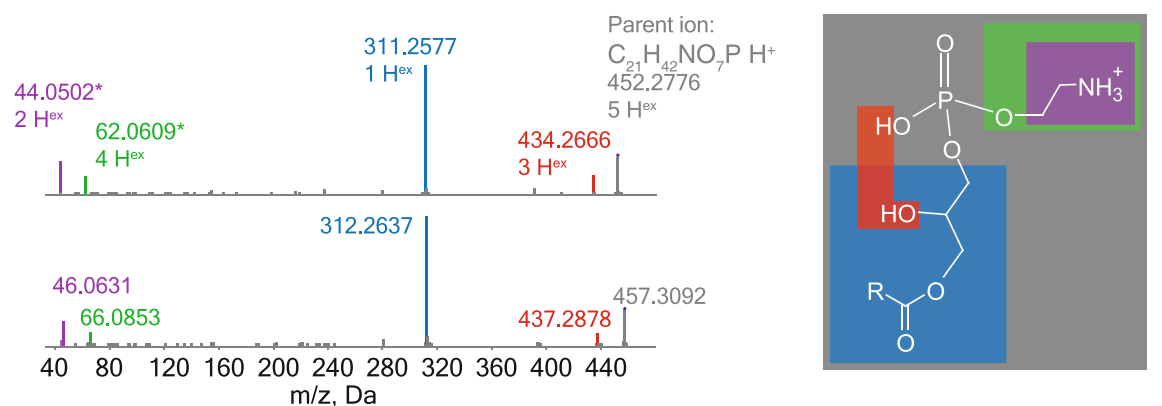

$\mathrm{m} / \mathrm{z}, \mathrm{Da}$

(c)

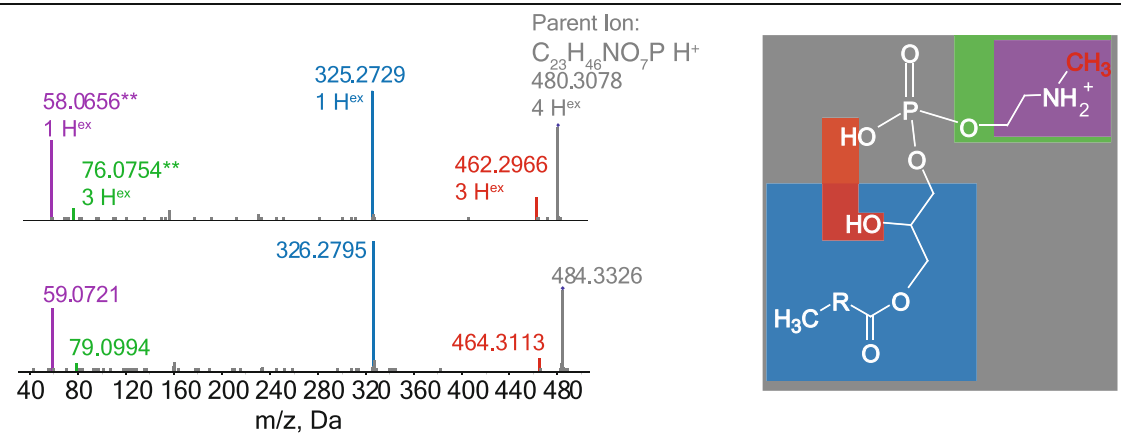

Fig. 3 Identification of intact polar lipid structures by $\mathrm{MS}^{2}$ and D exchange metabolomics. a Extracted ion chromatograms (EICs) for D exchange experiments carried out on two abundant acid mine drainage phospholipids (top). The feature with an identified chemical formula of $\mathrm{C}_{21} \mathrm{H}_{42} \mathrm{NO}_{7} \mathrm{PH}^{+}$, shows a strong EIC peak in D exchange experiments only at a mass corresponding to substitution of five deuterons for five protons; thus, the ion formula is $\mathrm{C}_{21} \mathrm{H}_{38} \mathrm{D}_{4} \mathrm{NO}_{7} \mathrm{D}^{+}$ (bottom). In contrast, the feature with an chemical formula of $\mathrm{C}_{23} \mathrm{H}_{46} \mathrm{NO}_{7} \mathrm{H}^{+}$has a strong D-exchanged EIC peak only at a mass corresponding to substitution of four deuterons for protons. Weaker peaks corresponding to 6 or 5 exchanged protons for the top and bottom panels, respectively, arise because of the natural abundance of ${ }^{13} \mathrm{C}$, the neutron of which cannot be distinguished from a ${ }^{2} \mathrm{H}$ neutron at $5 \mathrm{ppm}$ mass resolution. The EICs of typical, non-D-exchanged

contained one nitrogen atom. Comparison to authentic standards as shown in Supplemental Fig. S1 showed that these fragments corresponded to $N$-methyl ethanolamine moieties. The other $\mathrm{CH}_{3}$ substitution resulted in a mass shift of the 311 Da fragment of the 452 lipid to 325 Da for the 480 lipid. In both the 452 lipid and the 480 lipid, this fragment had only one exchangeable $H$, showing that the methylation was likely on the fatty acid substituent of the lyso $N$-monomethyl PE (lyso MMPE).

The features at $\mathrm{m} / \mathrm{z}=454$ and $\mathrm{m} / \mathrm{z}=482$ were analogous to the structures of the 452 and 480 lipids, respectively, except that the fragments at 311 and 325 Da were shifted to 313 and $327 \mathrm{Da}$, respectively, suggesting that lipids analyzed under the same conditions is shown, for clarity, on a negative scale. b MS ${ }^{2}$ fragmentation patterns of the $\mathrm{C}_{21} \mathrm{H}_{42} \mathrm{NO}_{7} \mathrm{PH}^{+}$ lipid under normal and D-exchanging conditions allows fragment-byfragment determination of exchangeable hydrogens and determination of molecular structure. Peaks marked with an asterisk were found to contain ${ }^{15} \mathrm{~N}$ during stable isotope labeling experiments and matched to $\mathrm{MS}^{2}$ peaks observed for an authentic ethanolamine standard. $\mathbf{c} \mathrm{MS}^{2}$ fragmentation patterns of the $\mathrm{C}_{23} \mathrm{H}_{46} \mathrm{NO}_{7} \mathrm{PH}^{+}$lipid under normal and D-exchanging conditions. Peaks marked with a double asterisk were found to contain ${ }^{15} \mathrm{~N}$ during stable isotope labeling experiments and matched to $\mathrm{MS}^{2}$ peaks observed for an authentic $N$-methyl ethanolamine standard. The color coding of peaks in $\mathbf{b}$ and $\mathbf{c}$ corresponds to the colored boxes on the proposed phospholipid structures (Color figure online)

these features were likely identical to 452 and 480 except for the saturation of the fatty acid side-chain. D exchange revealed that the $m / z=454$ lipid had five exchangeable hydrogens and the $\mathrm{m} / \mathrm{z} 482$ lipid had four, as shown in Supplemental Fig. S3. However, the $m / z=468$ feature had a more complex $\mathrm{MS}^{2}$ fragmentation spectrum, as shown in Supplemental Fig. S4. D exchange on this lipid could not distinguish whether the parent ion contained five exchangeable hydrogens or four. Supplemental Fig. S4 shows that this finding is due to hidden isomerism in the $m / z=468$ feature, which was found to be a mixture of lyso 17:0 PE (which has five exchangeably hydrogens) and lyso 16:0 MMPE (which has four). Similarly, the $\mathrm{m} / \mathrm{z}=466$ 


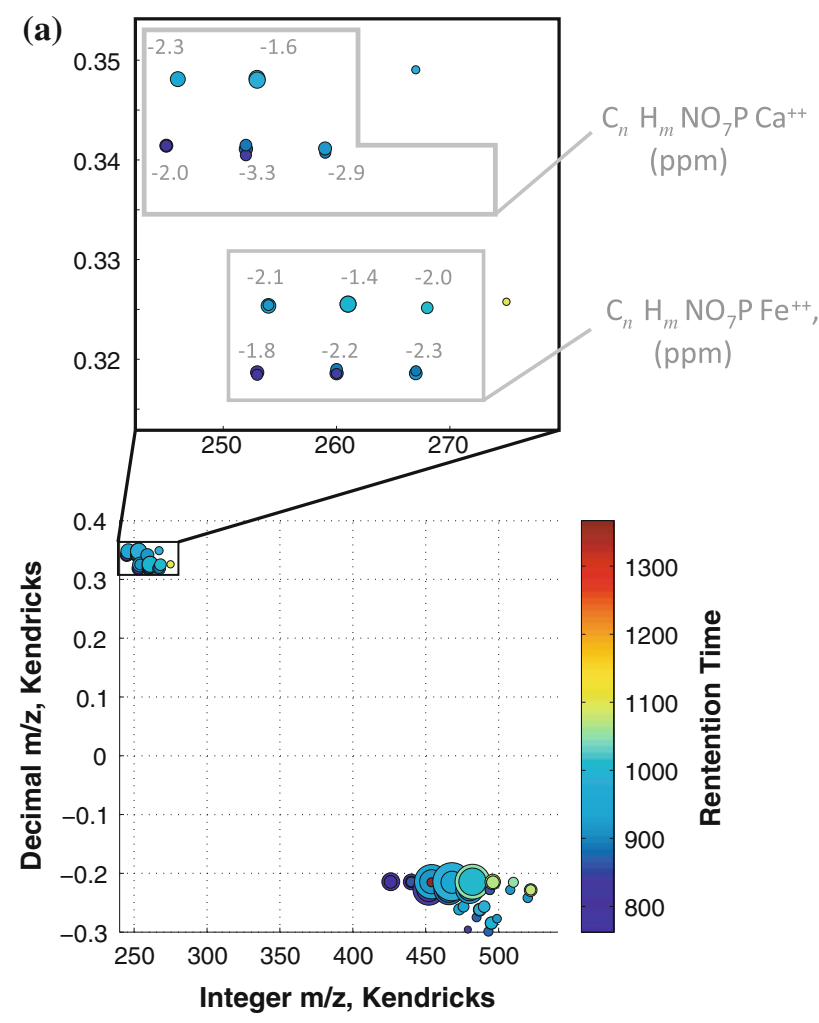

Fig. 4 Propensity of the lyso phospholipids for formation of adducts involving $\mathrm{Fe}$ and $\mathrm{Ca}$ cations. a Kendrick mass defect diagrams reveal a series of features that co-elute with lipid $\mathrm{MH}^{+}$ions with highly unusual mass defects. The accurate mass of these detected formulae is consistent with formulas of $\mathrm{C}_{n} \mathrm{H}_{m} \mathrm{NO}_{7} \mathrm{PCa}^{++}$and $\mathrm{C}_{n} \mathrm{H}_{m} \mathrm{NO}_{7} \mathrm{PFe}^{++}$.

feature was also found to be a mixture of isomers, corresponding to structures of lyso 17:1 PE and lyso 16:1 MMPE.

\subsection{Relationships between the PE lipids and other detected features}

The Kendrick mass defect diagram in Fig. 1b shows that small amounts of single-double bond equivalence (DBE) lyso phospholipids with fatty acid substituents as small as C14 were detected, as were small amounts of phospholipids up to $\mathrm{CH}_{2}$ heavier than the $\mathrm{C} 17: 1$ MMPE at $m / z=482.3241 \mathrm{Da}$. For the double DBE phospholipids, only the heavier phospholipid was found. However, these lipid variants differing by $\pm \mathrm{CH}_{2}$ units from the six most abundance lipid features had vastly reduced intensities than the six "core" lipids studied here.

Phosphatidylcholines (PCs) are $N$-trimethylated derivatives of PEs. They give rise to a characteristic neutral loss of $183 \mathrm{Da}$ as well as characteristic $\mathrm{MS}^{2}$ fragments $(\mathrm{m} / \mathrm{z}, 184.0729$ and 104.107). Features consistent with dimethylated and trimethylated (choline) forms of lipids are detected as minor features, as shown in SI Fig. 4. The 454.2928 feature shown in green is consistent with a lyso (b)

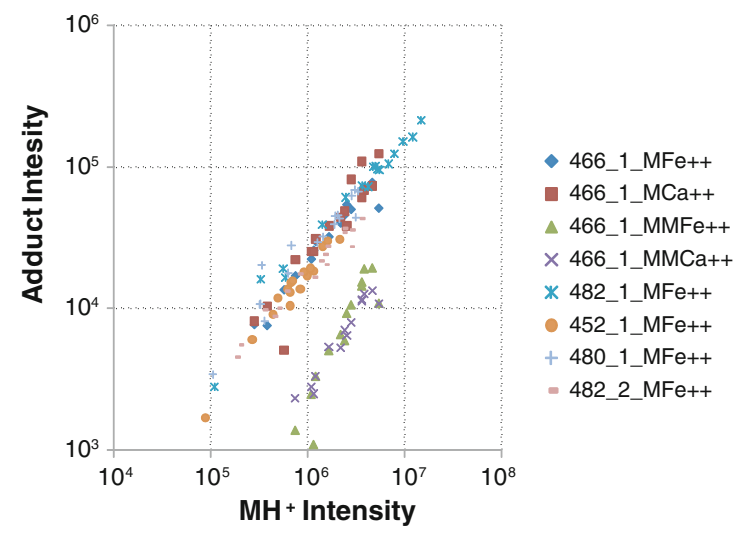

(c)

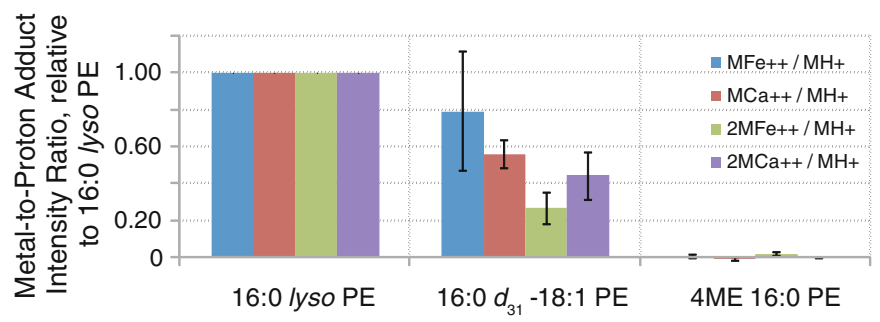

b The strong correlation of metal adduct intensity with $\mathrm{MH}^{+}$peak intensity suggests that the primary determinant of metal adduct abundance was lipid abundance. c Lyso lipids have a higher propensity to form iron and calcium adducts than do diacyl PE lipids

C14 dimethyl PE: it elutes earlier than longer-chain lipids and has 3 exchangeable hydrogens. The small blue peak corresponding to 2 exchangeable hydrogens for the 468.3085 and 482.3241 ions are consistent with the number of exchangeable hydrogens and chain lengths expected for lyso C14 and C15 PCs respectively. The spectral intensity of these features is lower than for the lyso MMPEs, indicating they are less abundant in the AMD biofilms.

Surprisingly, iron and calcium adducts of the lipids were observed. Figure 4 shows a Kendrick mass defect plot for features in the same retention time range as the detected lyso PE. The zoomed-in region of this plot shows the location of calcium and iron adducts more precisely. The adducts were within $4 \mathrm{ppm}$ or less of the predicted mass for adducts of the form $\mathrm{C}_{n} \mathrm{H}_{2 n} \mathrm{NO}_{7} \mathrm{PCa}^{++}$and $\mathrm{C}_{n} \mathrm{H}_{2 n} \mathrm{NO}_{7}$ $\mathrm{PFe}^{++}$. Dimeric adducts of the form $\left(\mathrm{C}_{n} \mathrm{H}_{2 n} \mathrm{NO}_{7} \mathrm{P}\right)_{2} \mathrm{Ca}^{++}$ and $\left(\mathrm{C}_{n} \mathrm{H}_{2 n} \mathrm{NO}_{7} \mathrm{P}\right)_{2} \mathrm{Fe}^{++}$were also detected for some lipids. In all cases, these adducts co-eluted with the corresponding $\mathrm{C}_{n} \mathrm{H}_{2 n} \mathrm{NO}_{7} \mathrm{PH}^{+}$molecular ion. The discovery of co-eluting metal adducts of this sort was unexpected because during chromatography, the lipids are initially strongly retained on the $\mathrm{C} 18$ column, while most salts are washed out of the column without binding. Thus, the lipid-metal adducts were stable throughout the chromatographic separation. 
Figure $4 \mathrm{~b}$ shows that across the 11 field samples studied (36 separate technical replicates), the intensity of the coeluting $\mathrm{MFe}^{++}, \mathrm{MCa}^{++}$, and $2 \mathrm{MFe}^{++}$adducts correlated strongly with the intensity of the protonated $\mathrm{MH}^{+}$ion, for all the lipid species detected.

Direct-infusion MS, with either regular or deuterated solvents, of $100 \mu \mathrm{M}$ solutions of pure lyso and diacyl PE standards incubated with $10 \mu \mathrm{M}$ of calcium or iron salts confirmed the formula assignments shown in Fig. 4a. These experiments also showed that the propensity of lyso lipids to form $\mathrm{Fe}$ or $\mathrm{Ca}$ adducts was higher for lyso PEs than for diacyl PEs (Fig. 4c). Interestingly, 1,2-diphytanoyl-sn-glycero-3-PE (4ME 16:0 PE), a PE bearing saturated, highly branched fatty acid substituents, had over 100 -fold reduced propensity to form $\mathrm{Fe}$ or $\mathrm{Ca}$ adducts compared to 18:0 lyso PE, while 1- $d_{31}$-palmitoyl-2-oleoyl$s n$-glycero-3-PE (16:0- $d_{31}$ 18:1 PE), a PE bearing one unbranched saturated and one unbranched mono-unsaturated fatty acid substituents, formed $\mathrm{Fe}$ and $\mathrm{Ca}$ adducts at a propensity between 27 and $79 \%$ of the wild-type. It is critical to note that we can not extrapolate these interesting results to the behavior of these lipids under aqueous equilibrium conditions much less their biological function. However, this observation could be tested using aqueousphase biochemical characterization of metal-lipid interactions though, these additional experiments go beyond the scope of the present study.

\subsection{Estimation of absolute abundance for lyso phospholipids}

Lyso PE lipids were highly abundant in methanol-chloroform extracts of lyophilized biomass as well as in the acidic water-alcohol extracts we used in our initial screening experiments. Also, addition of diacylated PE lipids as internal standards during extraction did not give rise to the corresponding lyso derivatives. Thus, we can rule out extraction artifacts as a source of the lyso lipids.

Absolute quantification of the 482 lipid, as estimated by $\mathrm{MS}^{2}$ peak intensity ratios between an isobaric internal standard and a natural sample, was $1.86 \pm 0.24 \mathrm{nmol} / \mathrm{mg}$ of lyophilized biomass, as shown in Supplemental Fig. S2. The other five lipids were between 0.42 and 2.16-fold more intense than the 482 lipid, and in total amounted to 6.16fold more $\mathrm{MS}^{1}$ intensity than the 482 lipid, suggesting that total lyso PE lipid abundances was on the order of $11 \mathrm{nmol} /$ $\mathrm{mg}$ dry biomass. In the bioreactor sample, a single-point estimation of the 482 lipid abundance gave $2.5 \mathrm{nmol} / \mathrm{mg}$ dry biomass, and the total intensity of all six features was 16.3-fold that of the 482 lipid alone, suggesting that the absolute abundance of all lyso PE lipids in the bioreactor was $40 \mathrm{nmol} / \mathrm{mg}$ dry biomass. For comparison, the diacyl PE content of Escherichia coli has been measured as
$33 \mathrm{nmol} / \mathrm{mg}$ of dry cell weight (Ames 1968). Thus, although our LC-MS technique did not detect diacyl PE lipids, we estimate that lyso PE lipids may account for the majority of PE lipids present in the AMD biofilm.

To reveal whether the lyso lipids are biosynthesized by cleavage of intact diacyl PE lipids, we spiked unlabeled diacyl PE lipids and unlabeled lyso C18:0 PE into live laboratory cultures of ${ }^{15} \mathrm{~N}$-labeled AMD biofilm. Because the polar nitrogen-containing head groups of natively synthesized PE lipids would be $0.997 \mathrm{Da}$ heavier than spiked lipids, we hoped to observe lyso PE biosynthesis from the exogenous diacyl PE lipids, but none was observed after $72 \mathrm{~h}$ of incubation (data not shown).

\subsection{Variation of lyso lipid abundance in natural samples}

Biofilms from the B Drift region of the Richmond Mine were visually assignable to DS based on thickness, an attribute shown to correlate with DS (Wilmes et al. 2009). At early DS0, biofilms are consistently dominated by the UBA-type Leptospirillum group II (Denef et al. 2010). This chemoautolithotroph is the first colonizer and primary producer of the biofilm community at the Richmond Mine (Goltsman et al. 2009; Tyson et al. 2004). At higher DS, the closely related Leptospirillum group II 5wayCG type becomes more prevalent, as do more divergent Leptospirillum lineages, fungi, and archaea.

Figure 5a shows the relative abundance of the six phospholipids in a co-located set of biofilm samples collected from the Richmond Mine in November 2007 (Wilmes et al. 2010). These samples were clearly distinguishable by DS. Mono-unsaturated phospholipids decrease in relative abundance at higher DSs, and monounsaturated fatty acid substituents increase. The trend is toward more saturated fatty acid side chains at higher DSs. The C16:1 lyso MMPE and C17:1 lyso PE co-elute. The C17:1 fatty acid substituent can be regarded as a formally methylated version of the C16:1 fatty acid substituent, meaning that both co-eluting features are mono-methylated. In contrast, the C16:1 PE and the C17:1 MMPE are unmethylated or dimethylated, respectively. A similar analysis holds for the saturated phospholipids. Grouping the phospholipids by the degree of methylation in this way reveals that unmethylated phospholipids are less abundant in more highly developed communities, while dimethylated phospholipids are more abundant. Monomethylated phospholipids had similar relative abundance at all DSs. The clustergram in Fig. 5b shows the normalized lipid abundance across a larger, more diverse series of samples from the Richmond Mine (Wilmes et al. 2010). Even across this larger sample set, phospholipid profiles distinguished 
Fig. 5 Variation of lyso PE and lyso MMPE in the environment. a Peak intensities of six lyso PE mass spectral features quantified by $\mathrm{MS}^{1}$ signal intensities and normalized to the total signal deriving from detected lyso PEs. Error bars indicate standard error in the mean for three independent technical replicates. "DS" developmental stage. b Biclustering analysis of the same six lyso PE mass spectral features across a more diverse, larger sample set. The panels on the left and top show the average Pearson correlationbased linkage distances between lipids and samples, respectively, while the heat map shows unitvector normalized phospholipid abundance. Above the sample labels is a grayscale indicator of the sample growth stage. An " $\mathrm{X}$ " label indicates samples whose growth stage was not classifiable due to profound differences in morphology and overall community structure (a)

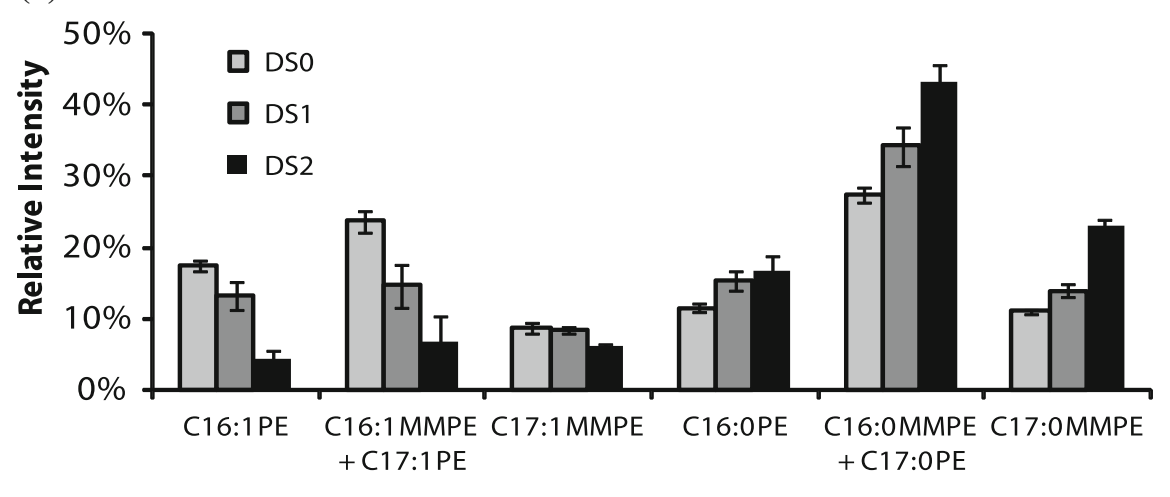

(b)
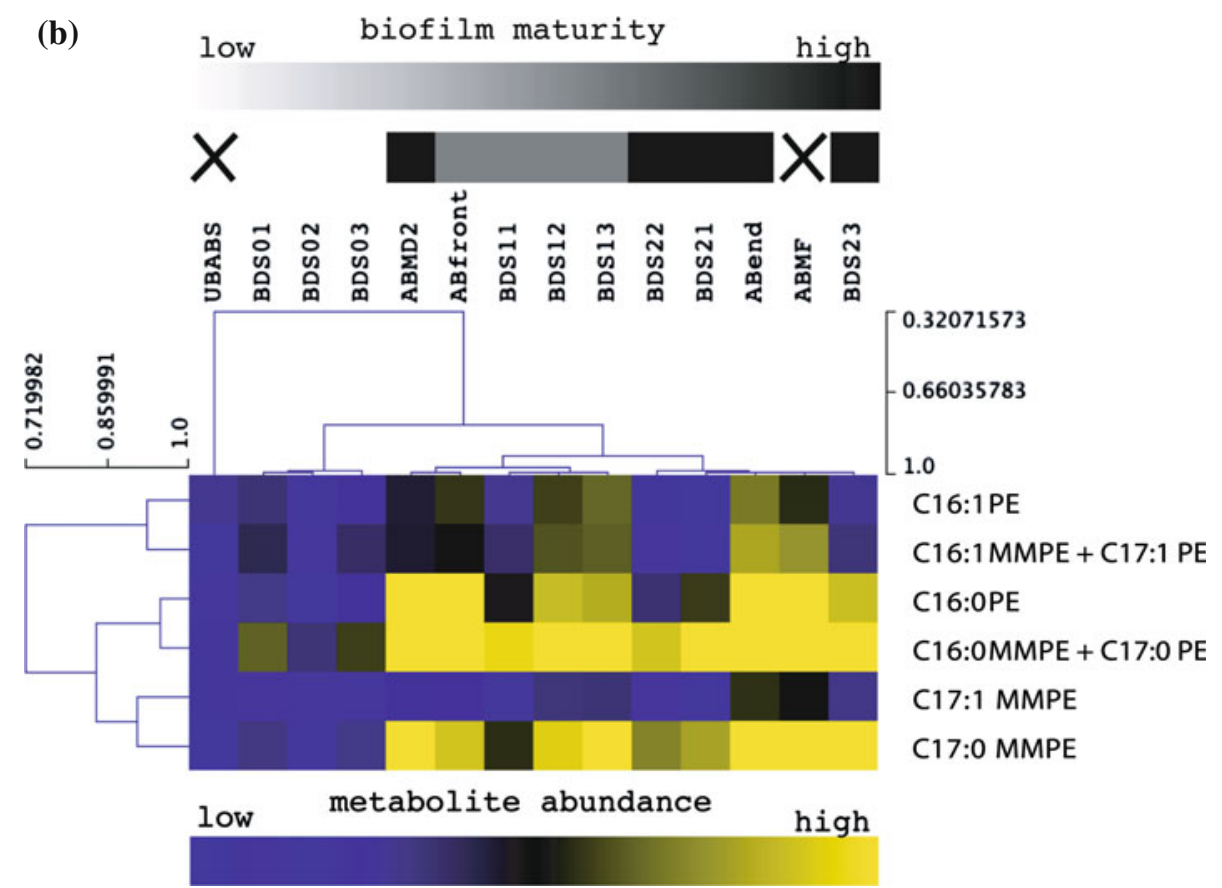

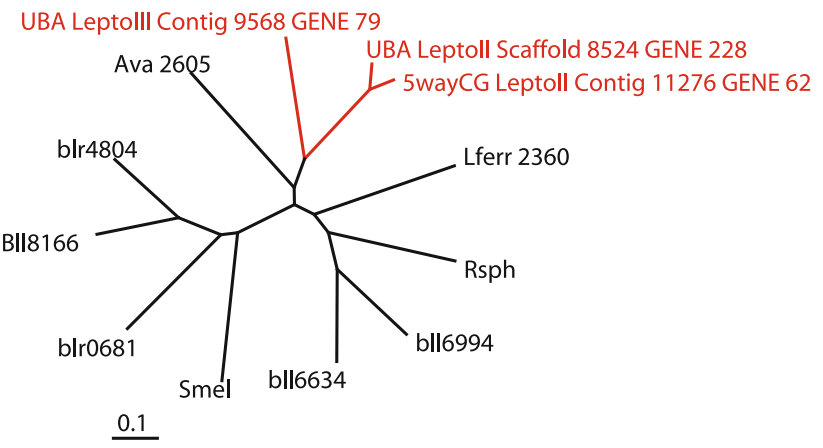

Fig. 6 Phylogenetic relationship between Leptospirillum methyltransferases and known PMTs. The unrooted tree was constructed by ClustalX and shows relationships between the indicated Leptospirillum ORFs (red) and known PMTs (black) from S. melliloti (SmeI), R. sphaeroides (Rsph), various isozymes from Bradyrhizobium japonicum (bll6994, bll6634, blr0681, blr4804, and B1l8166), A. ferrooxidans (Lferr 2360), and Anabaena variabilis (Color figure online) low DS biofilms (DS0) from higher stages of development (DS1 and DS2). The lyso PE and MMPE metabolite clustering shows that C16 lyso PE phospholipids have similar abundance profiles across the analyzed samples as their methylated C16 lyso MMPE + C17 lyso PE homologs. The same was not true of the lyso C17:1 and C17:0 lyso MMPE lipids.

\subsection{Bioinformatic and proteomic analysis of potential MMPE genes}

Bacteria that synthesize MMPEs are believed to do so through the action of PE $N$-methyltransferase (PMT) enzymes (EC 2.1.1.17, KEGG Orthology K00551). PMT enzymes can often multiply methylate PEs, with the trimethylated end product being PC. Two types of PMTs have been relatively well studied to date (Sohlenkamp 
et al. 2003): one type is represented by the protein encoded by pmtA from Sinorhizobium meliloti, and the other type represented by a protein encoded by pmtA from Rhodobacter sphaeroides (Sohlenkamp et al. 2003). On expression in $E$. coli, the $S$. meliloti enzyme, unlike the $R$. sphaeroides enzyme, led to accumulation of MMPE.

Blast searching and ClustalW alignment of an assembled metagenomic database derived from acid mine drainage communities against known PmtA proteins in the KEGG Orthology and NCBI nr databases, summarized in Supplemental Table S1, showed that three Leptospirillum open reading fragments (ORFs) share significant homology with known PMTs. These ORFs were previously annotated as "methyltransferase type 11 " or "putative methyltransferase". The phylogenetic relationship between these three genes and known PMT sequences is shown in Fig. 6 shows a phylogenetic tree of these sequences. The Leptospirillum sequences best match to a putative $N$-methyl transferase from Anabaena varabilis. They fall on a separate branch from the previously identified $R$. sphaeroides-like pmtA genes and the $S$. meliloti-like pmtA genes, suggesting that if these ORFs do encode PMT genes, they may be part of a new subfamily of this enzyme.

Only the putative PMT from UBA-type Leptospirillum group II (UBA_LeptoII_Scaffold_8524_GENE_228) enzymes has been detected in previous proteomics analyses (P. Wilmes and N. VerBerkmoes, unpublished data), where it was identified in a deep proteome sampling of the ABMF sample, which was shown in Fig. $5 b$ to have very high levels of MMPEs.

\subsection{Correlation network analysis}

A correlation network was constructed using previously generated proteomic and metabolomic data from a series of biofilm samples collected from Richmond Mine (Wilmes et al. 2010). The samples span a range of sampling times, geochemical conditions, and DSs (Wilmes et al. 2010). In the previous study, although distinct species-level protein and metabolite contingents were identifiable in the constructed correlation networks (e.g. differentiation of Leptospirillum group II- and III-associated small molecules), none of the metabolite features were structurally characterized. Furthermore, for the present network, we strain-resolved the Leptospirillum group II proteins, i.e. UBA-type proteins are apparent as purple triangles, whereas 5 way CG-type proteins are apparent as yellow triangles (Supplemental Fig. S5). We examined the network with a specific eye to potentially link the now-identified lyso PEs with specific proteins that were part of the community proteome (Supplemental Fig. S5). Network topology showed that the lyso PE metabolite abundances correlated only with UBA-type Leptospirillum type II proteins (purple triangles), in a tightly grouped, segregated cluster. This observation further suggested that the UBA-type Leptospirillum group II is solely involved in the biosynthesis of the lyso PEs.

\section{Discussion}

MS-based untargeted metabolomics seeks to identify mass spectral features on the basis of $\mathrm{MS}^{n}$ and accurate mass alone. Much work has gone into use of natural-abundance ${ }^{13} \mathrm{C}$ isotopologue peak intensities (Kind and Fiehn 2006, 2007) to identify empirical formulae. Such approaches are useful for identifying the chemical formula of detected features, but do not help identify chemical structures or resolve co-eluting isomers, unless coupled to extensive $\mathrm{MS}^{2}$ or $\mathrm{MS}^{3}$ fragmentation analysis.

Stable isotopic labeling in cell culture (Baran et al. 2010) can aid tremendously in determining empirical formulae, and when coupled to $\mathrm{MS}^{2}$ or $\mathrm{MS}^{3}$ fragmentation analysis, can reveal extensive structural information. However, the method requires the ability to completely isotopically label all of the $\mathrm{C}$ (or $\mathrm{N}$ or $\mathrm{H}$ or $\mathrm{O}$ ) atoms in a culture. This can be expensive or impossible for non-culturable organisms or entire ecosystems, or for experiments that would require prohibitively expensive isotopically labeled complex organic substrates.

The isotopic labeling technique used here enabled identification of comparatively complex lipid metabolites. Phospholipid fatty acids are usually analyzed by cleavage from their glycerol backbone and concomitant methanolysis, a process that separates the fatty acid moieties from attached head groups (Neufeld et al. 2007). Likewise, head groups have traditionally been cleaved from their attached acyl glycerols and analyzed by thin layer chromatography (Goldfine 1962; Goldfine and Hagen 1968; Shively and Benson 1967; Agate and Vishniac 1973). Electrospray techniques can be used to study intact polar lipids (Han and Gross 1996, 2005; Pulfer and Murphy 2003), but often an intact polar lipids analysis relies on the use of authentic standards or is limited to a known subset of bacterial lipids. The authors are not aware of any prior electrospray MSbased analysis of intact polar lipids that detected MMPEs.

The use of $\mathrm{D} / \mathrm{H}$ exchange allowed determination of sufficient metabolite structural information to identify intact polar lipids and to resolve closely related isomers not otherwise resolvable by MS or chromatography, as shown in Fig. 3. Similar isotope exchange techniques have been used in past metabolomics experiments (Liu et al. 2001, 2007; Lam and Ramanathan 2002). To lessen the amount of labeled $\mathrm{D}_{2} \mathrm{O}$ needed, these prior experiments often introduced $\mathrm{D}_{2} \mathrm{O}$ in the gas phase at the ion source, which results in less complete $\mathrm{D} / \mathrm{H}$ exchange than the method used in the current study (Fig. 2). The trend toward micro- 
and nano-scale flow rates in modern chromatography techniques makes the cost of label easily within reach of most investigators, even when using fully deuterated chromatography solvents.

Since $\mathrm{D} / \mathrm{H}$ exchange can be conducted after sample extraction, it may be a useful complement to traditional stable isotope labeling techniques, which require label addition prior to culture. As such, D/H exchange may prove to be a useful tool in the untargeted metabolomics studies of a broad range of systems.

The PE metabolites identified in this study contain three structural features that make them unusual relative to "classical" PEs: (i) $\mathrm{N}$-monomethylation, (ii), a lyso structure, meaning that the glycerol backbone contains a free hydroxyl group and only a single acyl substituent, and (iii) and fatty acid chains with an odd number of carbon atoms.

The MMPEs are intermediates in the biosynthesis of PCs by the PMT pathway, but many strains of bacteria that produce PC lipids contain minute or undetectable levels of MMPEs. In contrast, MMPE head groups have been identified as abundant in phospholipid fractions from some bacteria, including Clostridium butyricum (Goldfine and Howard 1962), Proteus vulgaris (Goldfine and Ellis 1964), S. meliloti (Sohlenkamp et al. 2003), and in three strains of Acidithiobacillus (Shively and Benson 1967; Short et al. 1969). Rather than simply being an intermediate in the synthesis of PC lipids, MMPE lipids may offer an advantage in extremely metals-rich, acidic environments. Support for this hypothesis includes findings that MMPEs are far less active than PEs as phase transfer catalysts for the transport of iron cations through hydrophobic phases (Agate and Vishniac 1972), and that phospholipids bind strongly to surfaces of pyrite (Zhang et al. 2003), a mineral commonly found in environments where Acidithiobacilli and Leptospirilla thrive. Thus, one possible role for MMPEs in Leptospirillum-dominated AMD biofilms may be to prevent uptake of toxic levels of iron cations. Further characterization of lipid-metal interactions under biologically relevant conditions will be required to address this hypothesis.

Lyso phospholipids have been identified in Halothiobacillus ssp. (Agate and Vishniac 1973), where their abundance increases upon the entry of a culture into late exponential or stationary phase. MMPE lipids were also identified in Halothiobacillus (Agate and Vishniac 1973), and their relative abundance increased in later growth stages. The pattern of increased lyso lipids and increased MMPE head groups in later growth stages is also seen in Fig. 5, which shows increased $N$-methylation (Fig. 5a) and increased abundance of lyso PE lipids relative to other metabolites (Fig. 5b). Like $N$-monomethylation, lysogenization of the lipid structure may provide for enhanced interaction with metal cations, as shown in Fig. 4. Like Leptosprillum and Acidothiobacillus ssp., Halothiobacilli live in high ionic-strength environments, although for Halothiobacillus the predominant electrolyte is sodium chloride, not iron(II) sulfate or sulfuric acid.

Phospholipids were estimated to represent $5 \%$ of the cell dry weight in Halothiobacillus, of which up to $8 \%$ were lyso lipids (Agate and Vishniac 1973). From these values and assuming an average phospholipid molecular weight of $700 \mathrm{Da}$, lyso phospholipid content of the culture can be estimated as $0.25 \mathrm{nmol} / \mathrm{mg}$ of dry weight. We measured a lyso lipid abundance of $10 \mathrm{nmol} / \mathrm{mg}$ wet weight in AMD biofilms. Weight loss during AMD biofilm lyophilization is routinely $75-80 \%$, which would mean that the lyso lipid abundance we detected would correspond to $40-50 \mathrm{nmol} /$ $\mathrm{mg}$ wet weight, far higher than previously detected in Halothiobacillus.

As stated above, the third unusual feature of the PEs identified in this study is the presence of odd-chain fatty acid esters. In prokaryotes, odd-chain fatty acids are often indicative of cyclopropanation (the formation of a cyclic propane group) or methenylation, modifications that can arise from methylation at or across the double bond of an unsaturated fatty acid. Cyclopropanation is a modification found commonly in many prokaryotes, especially under stress conditions. Cyclopropanation has been found to increase the acid tolerance of $E$. coli (Chang and Cronan 2002), and is a common modification found in Acidithiobacillus phospholipids (Cowie et al. 2009; Aston et al. 2009). Methenylation is found less commonly, and usually in eukaryote-associated organisms such as Mycobacterium ssp. (Buist 2007).

Several ORFs with significant homology to genes known to be involved in $N$-methyl PE biosynthesis were identified in the metagenome of the AMD community, but the complete pathway for lyso PE and $N$-methyl lyso PE biosynthesis remains poorly characterized. Our proteomics-coupled analysis (Supplemental Fig. S5) suggests that the UBA-type Leptospirillum group II may be particularly involved in the biosynthesis of these lipids.

Phospholipids found in the betaproteobacterial Acidithiobacillus genus share all three of the unique structural features $-N$-monomethylation, odd-chain fatty acids, and lyso structure-found in the lipids in this study. However, in contrast to Acidithiobacillus ssp., lipids from Leptospirillum ssp. of the Nitrospira phylum, which predominate in many AMD and bioleaching environments, have been less well studied. Although Leptospirillum and Acidithobacillus are phylogenetically distinct, they occupy very similar ecological niches. Both are aerobic, acidophilic chemoautolithotrophs that thrive in metals-rich environments. Interestingly, analogous structural features in their respective phospholipids may suggest a mechanism of convergent evolution for both Leptospirilla and Acidithobacilli. 
Acknowledgments The authors thank Mr. T. Arman for access to the Richmond Mine, Mr. R. Caver for on-site assistance, the Banfield lab members for assistance with biofilm sampling in the field, Susan Spaulding for assistance with bioreactor operation and sample preparation, and John Hayes for valuable technical discussions. This research was funded by the US Department of Energy, Office of Biological and Environmental Research Carbon-Cycling Program (DE-SC0004665), the DOE Genomics:GTL Program grant number DE-FG02-05ER64134. PWs recent involvement was supported by a Luxembourg National Research Fund ATTRACT grant (FNR/A09/ 03).

Conflicts of interests The authors declare no competing interests.

\section{References}

Agate, A. D., \& Vishniac, W. (1972). Iron transport by phospholipids in a two phase system containing water and $n$-pentanol. Chemistry and Physics of Lipids, 9(3), 247-254.

Agate, A. D., \& Vishniac, W. (1973). Changes in phospholipid composition of Thiobacillus neapolitanus during growth. Archives of Microbiology, 89(3), 247-255.

Allen, E. E., Tyson, G. W., Whitaker, R. J., Detter, J. C., Richardson, P. M., \& Banfield, J. F. (2007). Genome dynamics in a natural archaeal population. Proceedings of the National Academy of Sciences, 104(6), 1883.

Ames, G. F. (1968). Lipids of Salmonella typhimurium and Escherichia coli: Structure and metabolism. Journal of Bacteriology, 95(3), 833

Aston, J. E., Apel, W. A., Lee, B. D., \& Peyton, B. M. (2009). Toxicity of select organic acids to the slightly thermophilic acidophile Acidithiobacillus caldus. Environmental Toxicology and Chemistry, 28(2), 279-286.

Baker, B. J., Comolli, L. R., Dick, G. J., Hauser, L. J., Hyatt, D., Dill, B. D., et al. (2010). Enigmatic, ultrasmall, uncultivated Archaea. Proceedings of the National Academy of Sciences, 107(19), 8806.

Baran, R., Bowen, B. P., Bouskill, N. J., Brodie, E. L., Yannone, S. M., \& Northen, T. R. (2010). Metabolite identification in Synechococcus sp. PCC 7002 using untargeted stable isotope assisted metabolite profiling. Analytical Chemistry, 82(21), 9034-9042.

Belnap, C. P., Pan, C., VerBerkmoes, N. C., Power, M. E., Samatova, N. F., Carver, R. L., et al. (2010). Cultivation and quantitative proteomic analyses of acidophilic microbial communities. ISME Journal, 4(4), 520-530.

Buist, P. H. (2007). Exotic biomodification of fatty acids. Natural Product Reports, 24(5), 1110-1127.

Chang, Y. Y., \& Cronan, J. E. (2002). Membrane cyclopropane fatty acid content is a major factor in acid resistance of Escherichia coli. Molecular Microbiology, 33(2), 249-259.

Cowie, B. R., Slater, G. F., Bernier, L., \& Warren, L. A. (2009). Carbon isotope fractionation in phospholipid fatty acid biomarkers of bacteria and fungi native to an acid mine drainage lake. Organic Geochemistry, 40(9), 956-962.

Denef, V. J., Kalnejais, L. H., Mueller, R. S., Wilmes, P., Baker, B. J., Thomas, B. C., et al. (2010). Proteogenomic basis for ecological divergence of closely related bacteria in natural acidophilic microbial communities. Proceedings of the National Academy of Sciences of USA, 107(6), 2383-2390.

Goldfine, H. (1962). The characterization and biosynthesis of an $\mathrm{N}$-methylethanolamine phospholipid from Clostridium butyricum. Biochimica et Biophysica Acta, 59(2), 504-506.

Goldfine, H., \& Ellis, M. E. (1964). N-methyl groups in bacterial lipids. Journal of Bacteriology, 87(1), 8-15.
Goldfine, H., \& Hagen, P.-O. (1968). N-methyl groups in bacterial lipids III. Phospholipids of hypomicrobia. Journal of Bacteriology, 95(2), 367-375.

Goltsman, D. S. A., Denef, V. J., Singer, S. W., VerBerkmoes, N. C., Lefsrud, M., Mueller, R. S., et al. (2009). Community genomic and proteomic analyses of chemoautotrophic iron-oxidizing "Leptospirillum rubarum" (Group II) and "Leptospirillum ferrodiazotrophum" (Group III) bacteria in acid mine drainage biofilms. Applied and Environmental Microbiology, 75(13), 4599.

Han, X., \& Gross, R. W. (1996). Structural determination of lysophospholipid regioisomers by electrospray ionization tandem mass spectrometry. Journal of the American Chemical Society, 118(2), 451-457.

Han, X., \& Gross, R. W. (2005). Shotgun lipidomics: Electrospray ionization mass spectrometric analysis and quantitation of cellular lipidomes directly from crude extracts of biological samples. Bioorganic Chemistry, 24, 367-412.

Jeans, C., Singer, S. W., Chan, C. S., VerBerkmoes, N. C., Shah, M., Hettich, R. L., et al. (2008). Cytochrome 572 is a conspicuous membrane protein with iron oxidation activity purified directly from a natural acidophilic microbial community. The ISME Journal, 2(5), 542-550.

Kind, T., \& Fiehn, O. (2006). Metabolomic database annotations via query of elemental compositions: mass accuracy is insufficient even at less than 1 ppm. BMC Bioinformatics, 7, 234.

Kind, T., \& Fiehn, O. (2007). Seven Golden Rules for heuristic filtering of molecular formulas obtained by accurate mass spectrometry. BMC Bioinformatics, 8, 105.

Lam, W., \& Ramanathan, R. (2002). In electrospray ionization source hydrogen/deuterium exchange LC-MS and LC-MS/MS for characterization of metabolites. Journal of the American Society for Mass Spectrometry, 13(4), 345-353.

Liu, D. Q., Hop, C. E. C. A., Beconi, M. G., Mao, A., \& Chiu, S. H. L. (2001). Use of on-line hydrogen/deuterium exchange to facilitate metabolite identification. Rapid Communications in Mass Spectrometry, 15(19), 1832-1839.

Liu, D. Q., Wu, L., Sun, M., \& MacGregor, P. A. (2007). On-line H/D exchange LC-MS strategy for structural elucidation of pharmaceutical impurities. Journal of Pharmaceutical and Biomedical Analysis, 44(2), 320-329.

Lo, I., Denef, V. J., Verberkmoes, N. C., Shah, M. B., Goltsman, D., DiBartolo, G., et al. (2007). Strain-resolved community proteomics reveals recombining genomes of acidophilic bacteria. Nature, 446(7135), 537-541.

Mueller, R. S., Denef, V. J., Kalnejais, L. H., Suttle, K. B., Thomas, B. C., Wilmes, P., Smith, R. L., Nordstrom, D. K., McCleskey, R. B., Shah, M. B., VerBerkmoes, N. C., Hettich, R. L., \& Banfield, J. F. (2010). Ecological distribution and population physiology defined by proteomics in a natural microbial community. Molecular Systems Biology, 6, 314.

Neufeld, J. D., Wagner, M., \& Murrell, J. C. (2007). Who eats what, where and when? Isotope-labelling experiments are coming of age. The ISME Journal, 1(2), 103-110.

Pulfer, M., \& Murphy, R. C. (2003). Electrospray mass spectrometry of phospholipids. Mass Spectrometry Reviews, 22(5), 332-364.

Ram, R. J., Verberkmoes, N. C., Thelen, M. P., Tyson, G. W., Baker, B. J., Blake, R. C., et al. (2005). Community proteomics of a natural microbial biofilm. Science, 308(5730), 1915-1920.

Shively, J. M., \& Benson, A. A. (1967). Phospholipids of Thiobacillus thiooxidans. Journal of Bacteriology, 94(5), 1679.

Short, S. A., White, D. C., \& Aleem, M. I. H. (1969). Phospholipid metabolism in Ferrobacillus ferrooxidans. Journal of Bacteriology, 99(1), 142.

Singer, S. W., Chan, C. S., Zemla, A., VerBerkmoes, N. C., Hwang, M., Hettich, R. L., et al. (2008). Characterization of cytochrome 
579, an unusual cytochrome isolated from an iron-oxidizing microbial community. Applied and Environmental Microbiology, 74(14), 4454.

Sohlenkamp, C., López-Lara, I. M., \& Geiger, O. (2003). Biosynthesis of phosphatidylcholine in bacteria. Progress in Lipid Research, 42(2), 115-162.

Tyson, G. W., Chapman, J., Hugenholtz, P., Allen, E. E., Ram, R. J., Richardson, P. M., et al. (2004). Community structure and metabolism through reconstruction of microbial genomes from the environment. Nature, 428(6978), 37-43.

Wilmes, P., Remis, J. P., Hwang, M., Auer, M., Thelen, M. P., \& Banfield, J. F. (2009). Natural acidophilic biofilm communities reflect distinct organismal and functional organization. The ISME Journal, 3(2), 266-270.
Wilmes, P., Bowen, B. P., Thomas, B. C., Mueller, R. S., Denef, V. J., VerBerkmoes, N. C., et al. (2010). Metabolome-proteome differentiation coupled to microbial divergence. mBio, 1(5), e00246.

Zhang, X., Borda, M. J., Schoonen, M. A. A., \& Strongin, D. R. (2003). Adsorption of phospholipids on pyrite and their effect on surface oxidation. Langmuir, 19(21), 8787-8792. 